\title{
Scaffold Analysis of Ligands Exhibiting GPCR Signaling Bias
}

\author{
Jason E. Sanchez, ${ }^{\dagger}$ Govinda KC, ${ }^{\dagger}$ Julian Franco, ${ }^{\ddagger}$ and Suman Sirimulla*,,$\dagger$ \\ †Computational Science Program, The University of Texas at El Paso, Texas, 79968, USA \\ $\ddagger$ Mechanical Engineering, The University of Texas at El Paso, Texas, 79968, USA \\ ФDepartment of Pharmaceutical Science, The University of Texas at El Paso, Texas 79968, \\ USA. \\ E-mail: ssirimulla@utep.edu \\ Phone: (915) 747-8530
}

\begin{abstract}
Signaling bias is a feature of many G-protein coupled receptor (GPCR) modulating drugs with clinical implications. Whether it is therapeutically advantageous for a drug to be G Protein biased or $\beta$-Arrestin ( $\beta$-Arr) biased, depends on the context of the signaling pathway. Here, we explored GPCR ligands that exhibit biased signaling to gain insights into scaffolds and pharmacophores that leads to bias. More specifically, we used BiasDB, a database containing information about GPCR biased ligands and all ligands which show a $(\beta$-Arr $) / G$ protein bias or a $G$ protein / $\beta$-Arr bias are considered for the study. Four machine learning models were trained on these ligands to classify them. The features which were most important for training the models were analyzed. Two of these features (number of secondary amines and number of aromatic amines) were more prevalent in $\beta$-Arr biased ligands. After training a Random Forest model on HierS scaffolds, we found five scaffolds which demonstrated G protein or $\beta$-Arr bias. We also conducted t-SNE clustering, observing correspondence between
\end{abstract}


unsupervised and supervised machine learning methods. To increase the applicability of our work, we developed a web implementation of our models which can predict bias based on a user-provided SMILES patterns. Our web implementation is available at: drugdiscovery.utep.edu/biasnet.

\section{Introduction}

G-protein coupled receptors (GPCRs) represent the largest category of druggable targets for FDA approved drugs - approximately 34 percent of all drugs. ${ }^{1,2}$ GPCRs are signal transducing proteins that relay information from the extracellular environment of a cell to the cytosol. Upon binding to a drug molecule, GPCRs undergo a conformational change leading an associated G protein to exchange GDP for GTP. Once this exchange occurs, G proteins are activated and propagate the signaling cascade, ultimately resulting in a cellular response.

$\mathrm{G}$ proteins are composed of three subunits: $\mathrm{G} \alpha, \mathrm{G} \beta$, and $\mathrm{G} \gamma$. Among these subunits, $\mathrm{G} \alpha$ - the subunit most often responsible for eliciting the cellular/drug response -is categorized into one of four families: Gs, Gi/o, Gq/11 or G12/13. ${ }^{3}$ Each of these four families activates a specific set of second messengers. For example, Gs stimulates the production of cAMP. In addition to activating $\mathrm{G}$ proteins, a GPCR may also activate $\beta$-Arr. ${ }^{4}$ The preferential activation of either the G protein, $\beta$-Arr is termed biased signaling.

In spite of the extensive druggability of GPCRs there remains much to be learned about GPCR signaling - both among known and potential GPCR ligands. Signaling bias, a phenomenon first described in 1995, is feature with the potential of influencing the discovery and approval of novel pharmaceutical agents. ${ }^{5}$ Because drug molecules targeting a specific signaling pathway minimize the risk of side effects, developing biased drugs is in the public interest. In light of this need, many studies have been conducted to characterize cases of GPCR signaling bias.

BiasDB is a manually curated, database of such studies. ${ }^{6}$ BiasDB features 654 cases of biased signaling organized into 4 bias categories: G protein, G protein selective, Extracellular 
Signal-Regulated Kinases (ERK), and $\beta$-Arrestin ( $\beta$-Arr). In addition to compiling known cases of signaling bias, BiasDB also provides a preliminary analysis of the chemical features that tend to make a molecule biased. Knowing these general features can provide a good starting point for drug discovery scientists to develop biased molecules; however, a more detailed Structure Activity Relationship (SAR) is needed.

Knowing this, our research objective was twofold. First, we used BiasDB to train machine learning models to classify an unknown molecule as a $\mathrm{G}$ protein biased ligand or $\beta$-arr biased ligand to observe if there were global patterns of bias found for many receptor types. To this end we used Random Forrest (RF), Multi-layer Perception (MLP), XGBoost (XGB), and Directed Message Passing Neural Network (DMPNN). We then validated our four models and compared their performance. Next, we determined the ten most important RDKit features to understand how the machine learning models were best able to classify the ligands. We noticed two molecular fragments - the number of secondary amines, and number of nitrogens - were a part of these features. Knowing this, we compared these fragments for G protein biased ligands and $\beta$-arr biased ligands. We then provided examples of biased and unbiased ligands to show how these features may explain the SAR of the biased molecules. Finally, we retrained a RF model using chemical scaffolds generated by the HierS method. ${ }^{7}$ Using these scaffolds as features, we determined which scaffolds are most important for classifying ligands. Five scaffolds which show a bias for either $\beta$-arrestin or $\mathrm{G}$ proteins are presented here. We conclude with a discussion of these scaffolds as they relate to three biased drugs.

\section{Results and Discussion}

Machine learning test metrics for each of our four models are shown in Figure 1. Receiver Operator Characteristic Area Under the Curve (ROC AUC) is a popular metric by machine learning models are often evaluated. For each of our models the ROC AUC was above 0.8 , which is generally considered excellent. ${ }^{8}$ The performance of our models for each of 
the remaining metrics is as follows. All of our models scored an accuracy above 0.8. The F1 score, which takes into account recall and precision, for all of our models was above 0.7. Sensitivity for our models was greater than 0.7 and for two of our models (MLP and DMPNN) this number was 0.8. The specificity of our models was above 0.8 and for two of our models (XGB and RF) this number was above 0.9. Lastly, the cohen kappa values for each of our models was above 0.6 except for DMPNN which had a value of 0.599 . McNemar Test results (Table 1) show there is no statistically significant difference between our four models (all pairwise p-values are $>0.05$ ). Based on these results, we conclude our models were able to distinguish $\beta$-arr biased ligands from $\mathrm{G}$ protein biased ligands in our training set. Additionally, we observe there was no statistically significant difference between our models' abilities to distinguish $\mathrm{G}$ protein biased ligands from $\beta$-arr biased ligands.

Table 1: Pairwise McNemar Test p-values

\begin{tabular}{|l|l|l|l|l|}
\hline & MLP & XGB & RF & DMPNN \\
\hline MLP & NA & 1 & 1 & 0.727 \\
\hline XGB & 1 & NA & 1 & 0.774 \\
\hline RF & 1 & 1 & NA & 0.754 \\
\hline DMPNN & 0.727 & 0.774 & 0.754 & NA \\
\hline
\end{tabular}

From our models, we determined the number of secondary amines was the most important fragment in determining bias. Ligands in BiasDB had anywhere from 0 to 14 secondary amines. Figure 2 shows $\beta$-arr biased ligands tend to have more secondary amines than $\mathrm{G}$ protein biased ligands. Chi-Squared test $(\mathrm{p}$-value $<0.01)$ also demonstrate that there is a statistically significant difference between the number of $\beta$-arr biased secondary amines and $\mathrm{G}$ protein biased secondary amines. These statistics suggests increased secondary amines are connected to $\beta$-arrestin bias relative to $\mathrm{G}$ protein bias in the BiasDB database.

To make sense of these data, we chose to consider the two molecules in Figure 3. Both molecules are ethanolamines and agonists for the $\beta-2$ receptor and presumably have a similar mechanism of action. The molecule on the left (isoproterenol) contains only one secondary amine while the molecule on the right (BI-167107) contains two secondary amines. The 
Test set results

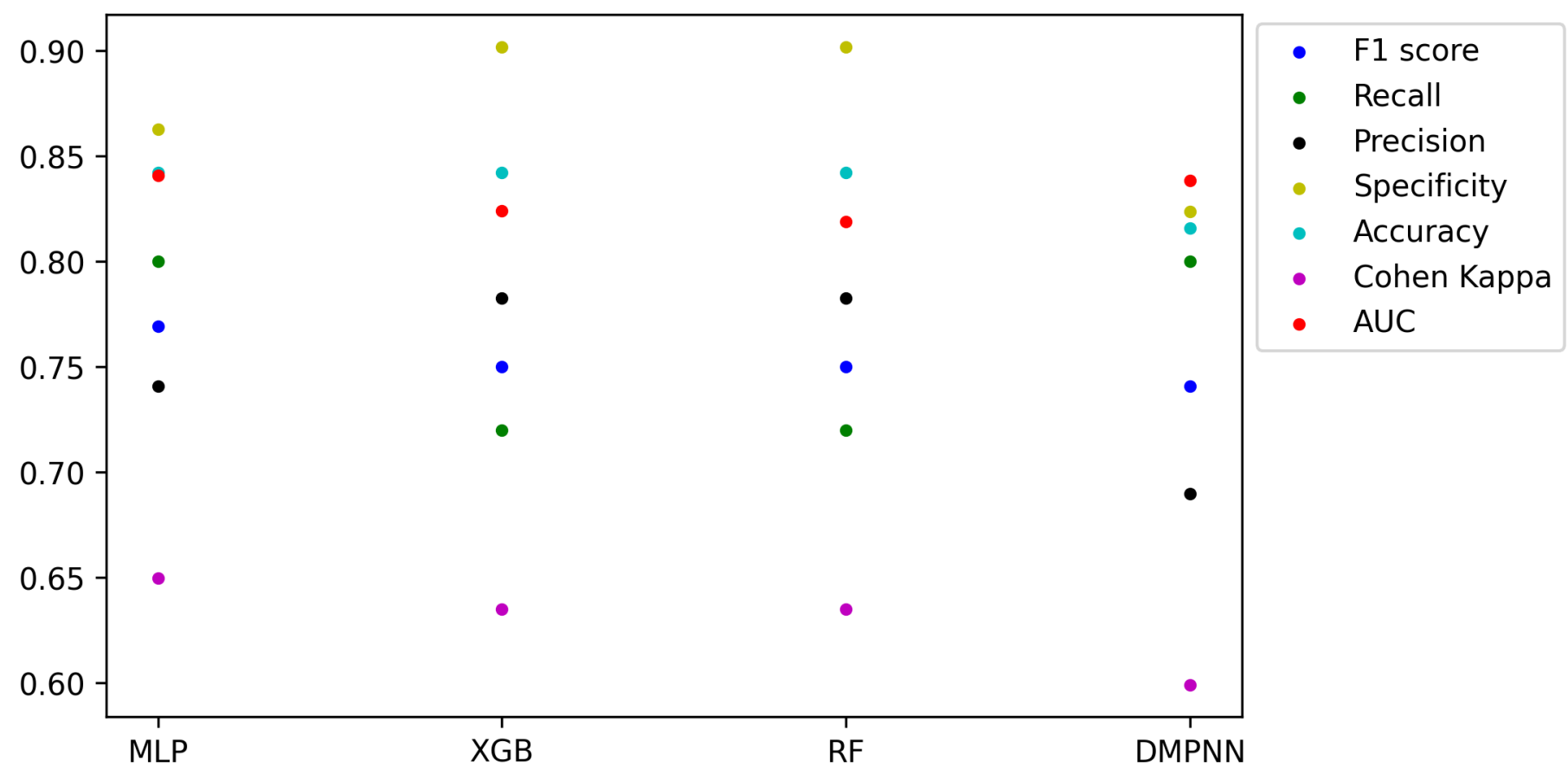

Figure 1: Scatter plot showing the performances of the RF, XGB, MLP, and DMPNN
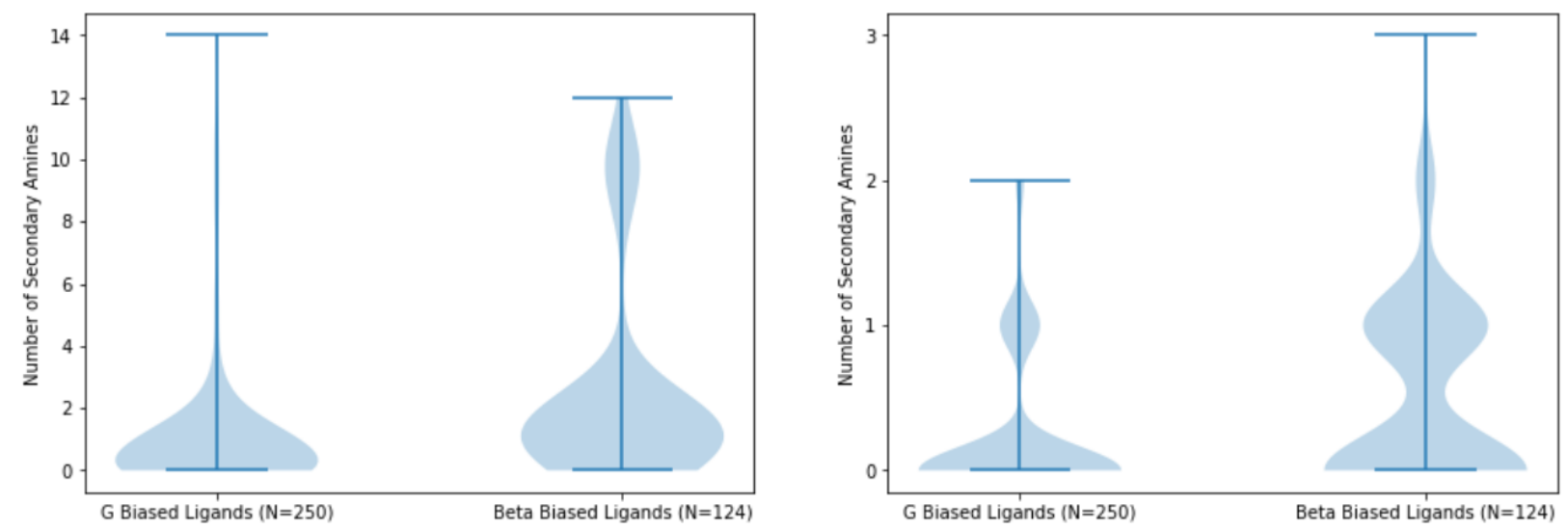

Figure 2: Violin Plot Comparing RDKit Fragments for G protein biased ligands and $\beta$-arr biased ligands

molecule on the left is unbiased while the molecule on the right is $\beta$-arrestin biased.

Previous docking studies have demonstrated isoproterenol forms hydrogen bonds between the para hydroxyl oxygen and SER 204 on Transmembrane 5 (TM5). ${ }^{9}$ Additionally the compound forms a salt bridge between its only nitrogen atom and ASP 113 on Transmembrane 3 (TM3). 


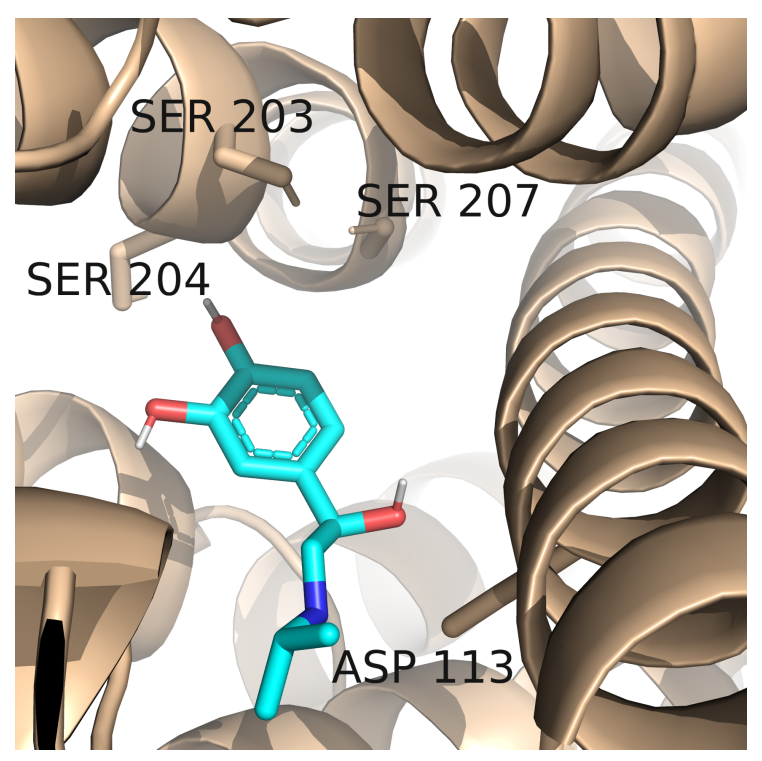

(a) Isoproterenol

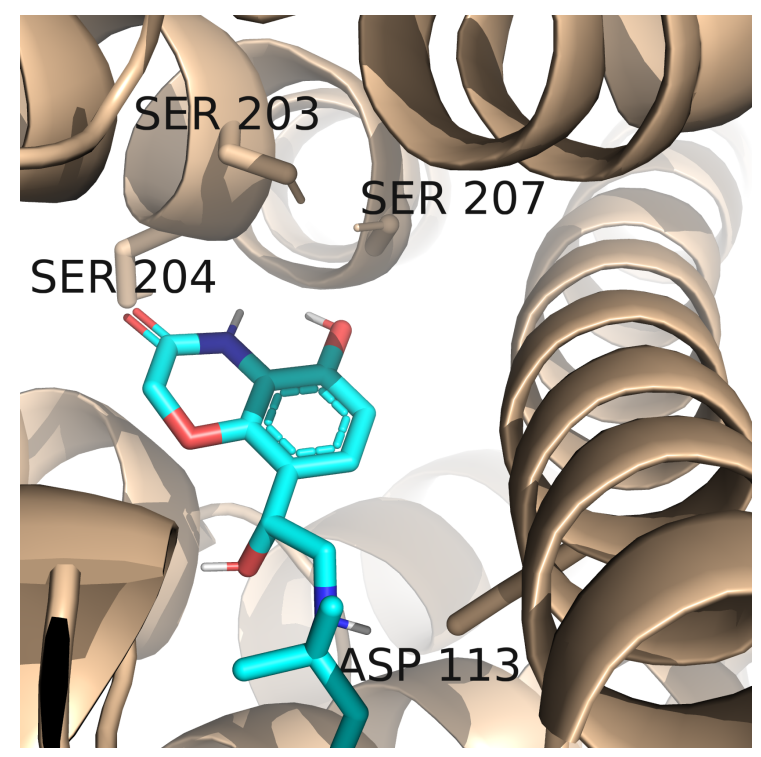

(b) BI-167107

Figure 3: Molecular Docking Simulations to the $\beta-2$ Adrenergic Receptor

We performed docking studies to compare the binding of isoproterenol and BI-1607107 to the $\beta-2$ Adrenergic Receptor. These results are shown in Figure 3. Like in the study by Weiss and colleagues, the para-hydroxyl group on isoproterenol appears in close proximity to serine 203 and serine 204 (Figure 3a). BI-1607107 shows a similar proximity to serine 203 and serine 204 with a secondary ring-member amine (Figure 3b). This proximity leads us to believe BI-1607107 has the capacity to form a hydrogen bonds to a serine residue in TM5 like isoproterenol.

An important difference to note is that the ring nitrogen in BI-167107 is a less potent hydrogen bond donor than isoproterenol. We believe the stronger interaction of isoproterenol with TM5 might account for the balanced bias demonstrated by isoproterenol. By the same reasoning, the weaker interactions between BI-167107 might explain $\beta$-arrestin bias. In either case, crystal structure experiments elucidating the role of serine 203, serine 204, and serine 207 are needed to confirm our hypothesis about $\beta$-arrestin bias.

The number of aromatic amines was the second most important fragment in training our models. The number of aromatic nitrogen atoms for $\mathrm{G}$ protein biased ligands and $\beta$-arr biased ligands ranged from 0 to 4 . A table of total counts for our $\beta$-arr biased ligands and $\mathrm{G}$ 
protein biased ligands is shown in Table 4 . Figure 2 shows $\beta$-arr biased ligands tend to have more aromatic nitrogen atoms than $\mathrm{G}$ protein biased ligands. Chi-square testing (p-value $<$ 0.01) also demonstrate that there is a statistically significant difference between the number of $\beta$-arr biased ligand and $\mathrm{G}$ protein biased ligand aromatic nitrogen atoms.

We explored two compounds to get a sense of how aromatic nitrogens atoms could influence bias. Figure 4 shows the unbiased reference compound, quinpirole, and a biased compound 5-Hydroxy-8-(4-methylpiperazin-1-yl)-4H-1,4-benzoxazin-3-one for the D2 receptor. The former compound has an immidazole ring and no aromatic amines. The latter compound has a phenol ring with an aromatic amine at a para position to the phenol hydroxyl. We suspect the adjacent position of the amine and the aromatic ring in 5-Hydroxy-8-(4methylpiperazin-1-yl)-4H-1,4-benzoxazin-3-one is important to determining bias.

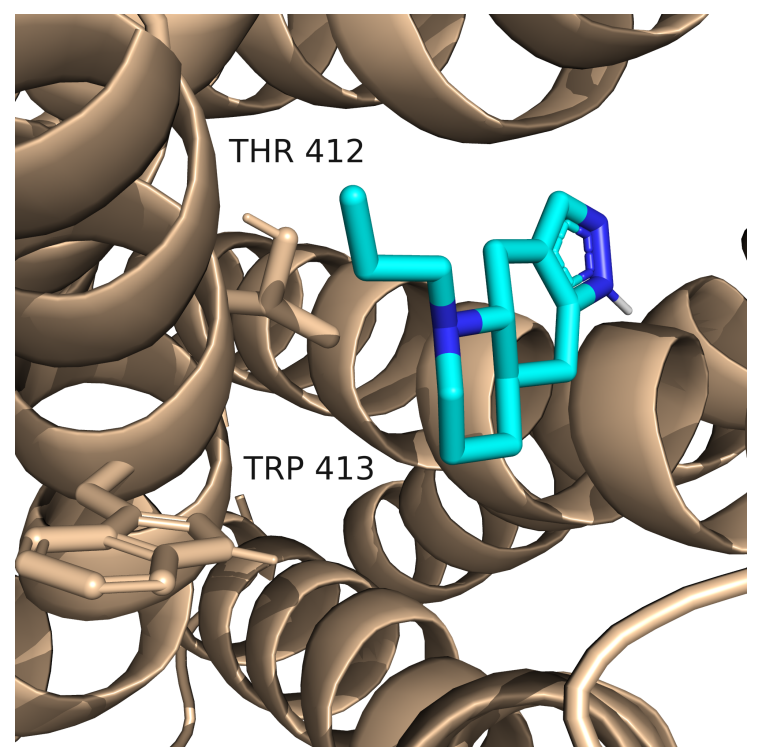

(a) Quinpirole

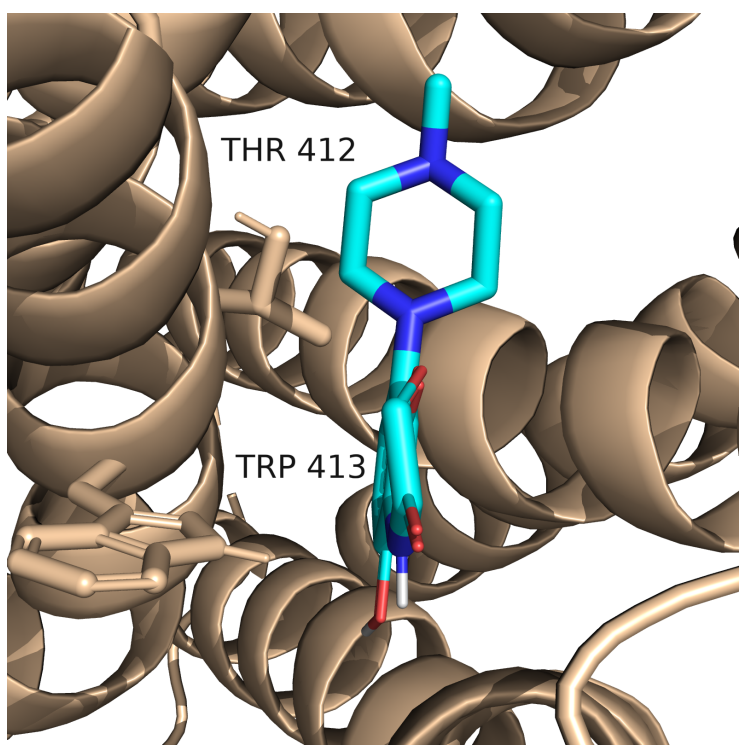

(b) 5-Hydroxy-8-(4-methylpiperazin-1-yl)-4H-1,4benzoxazin-3-one

Figure 4: Molecular Docking Simulations to the D2 Receptor

Previous mutagenesis studies suggest Transmembrane 3 (TM3) is key to determining functional selectivity for the D2 receptor. ${ }^{10}$ Work by Peterson and colleagues showed mutations on TM3 proximal to the GPCR/G Protein interface resulted in $\beta$-arrestin bias while mutations distal to the same interface resulted in G-Protein selectivity. Although a complete 
characterization of how these mutations leads to bias is not discussed, we believe increasing binding between interactions between ligands and TM3 result in greater bias. Indeed, functional selectivity studies on the D2 receptor show Asp 3.32 forms salt-bridge interactions between the residue and ligands with an ionizable nitrogen. ${ }^{11}$ These interactions are thought to be important to maintaining G Protein bias.

Based on this logic, interactions which serve to distance ligands from TM3 - particularly those that prevent binding to ASP 3.32 - may explain $\beta$-arrestin bias. We performed docking studies to show the difference in binding for quinpirole and 5-Hydroxy-8-(4-methylpiperazin1-yl)-4H-1,4-benzoxazin-3-one against the D2 receptor. The results are shown in Figure 4. Quinpirole is shown with its aromatic moiety facing toward ASP 114 (on TM3). The aromatic nitrogen is oriented in such a way to suggest hydrogen bonding is possible between the ligand and ASP 114. We believe this conformation is consistent with G Protein bias at the D2 receptor.

The biased compound 5-Hydroxy-8-(4-methylpiperazin-1-yl)-4H-1,4-benzoxazin-3-one, does not exhibit the same hydrogen bond as quinpirole. Instead, the molecule is oriented in such a way that its tertiary amine forms hydrogen bonds with THR 412. Additionally, the aromatic ring itself appears to form $\pi-\pi$ interactions with TRP 413. Together the aromatic ring and the tertiary amine on 5-Hydroxy-8-(4-methylpiperazin-1-yl)-4H-1,4-benzoxazin-3-one orient the aromatic amine away from ASP 114 and distance the molecule from TM3.

Because this aromatic amine is the only ionizable atom, on 5-Hydroxy-8-(4-methylpiperazin1-yl)-4H-1,4-benzoxazin-3-one the molecule has no other atom with which to from a salt bridge, or hydrogen bond with ASP 114.

Because aromatic amines are, by definition, adjacent to aromatic moieties, the potential of these amines to form hydrogen bonds, ion-ion interactions, or ion-dipole interactions can be limited by the adjacent aromatic moieties. We suspect that in BiasDB such effects account for the $\beta$-arrestin bias exhibited by many molecules. Moreover, we believe our machine learning models detected aromatic amines as an important fragment because similar patterns may be 
observed with other molecules.

In addition to considering molecular features, our goal was also to utilize machine learning models trained on scaffolds. The top five scaffolds found in our models are discussed here.

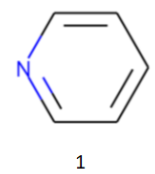

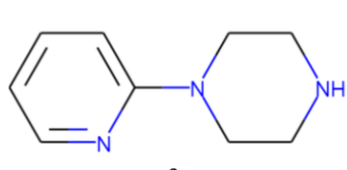

2

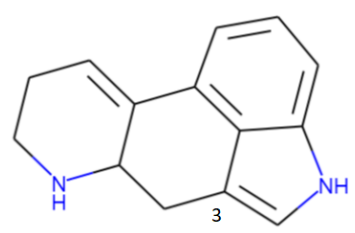

Figure 5: Biased Scaffolds
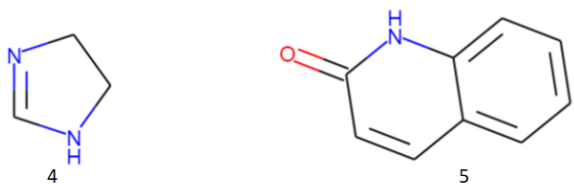

Scaffold 1 is the molecule pyridine. There are 123 cases of bias on BiasDB with the pyridine substructure. Of these cases, 79 are $\mathrm{G}$ protein biased and only 13 are $\beta$-Arrestin bias. Scaffold 1 is overwhelmingly present in cases of $\mathrm{G}$ protein bias.

Scaffold 1 exhibits a considerable degree of receptor diversity. The pyridine structure is found in $\mathrm{G}$ protein bias cases involving 10 different receptors (5-HT1, 5-HT2, CB2, CXCR3, D1, D2, Ghrelin, LH, Kappa, and Mu receptor).

Scaffold 1 is a sub-scaffold of scaffold two. Unsurprisingly, scaffold 2 is also heavily represented in G protein bias cases. However, scaffold 2 has less receptor diversity than scaffold 1. In fact, scaffold 2 represented only 5HT1 and D1 ligands. This seems to suggest that certain structural features of pyridine help contribute to general G protein bias. However, structural features of pyridine bonded with piperazine (scaffold 2) contribute to more specific instances of bias.

Two of the most salient features of pyridine are the basic nitrogen which acts as a hydrogen bond acceptor and the aromatic ring which confers planar molecular geometry to the scaffold. The planar conformation and nitrogen lone pair might are important for binding to many classes of $\mathrm{G}$ protein receptors may account for bias. We explore examples in the literature which support this idea.

The D1 receptor natively binds catecholamines. However, there exists examples of pyridine-containing non-catecholamines binding to the D1 receptor. ${ }^{12}$ These molecules con- 
tain scaffold 1 fused to an imidizole. Together these features impart the fused ring system with a planar shape that allows the molecule to fit into the binding pocket of D1. The nitrogen on the imidizole interacts with SER 188. The hydrocarbon skeleton of the ring system interacts with LEU 190. In both these interaction the "slender" molecular geometry characteristic of scaffold 1 is necessary for bias.

Scaffold 1 was also present for both Kappa and Mu opioid receptors. In fact, other studies have already developed scaffolds for Kappa and $\mathrm{Mu}$ receptors using pyridine as a sub-scaffold in their larger super-scaffold structures. ${ }^{13,14}$ These super scaffolds are termed triazole analogs and 2-Pyridyl Spirocyclic core molecules. For both of these super-scaffolds the pyrimidine structure was not modified in any way scaffold 1 necessary for G protein selectivity.

We finish our discussion of scaffold 1 by addressing a lack of information on the SAR of pyridine for the remaining receptors. Even thought there does not currently exist literature tying scaffold 1 to $\mathrm{G}$ protein bias for A2B, CB2, CXCR3, Ghrelin, and LH receptors there remains the possibility that such a bias does exist. More work on the SAR underlying signaling bias for these receptors needs to be done to confirm if pyridine does play an important role in general $\mathrm{G}$ protein biased signaling.

Scaffold 2, 1-(2-Pyridinyl)piperazine, is a super-scaffold containing pyridine (scaffold 1). There are 17 cases of bias on BiasDB with the scaffold 2 substructure. Of these cases, 1 case is an ERK bias and 16 cases are G protein bias. Taken together these numbers suggest Scaffold 2 strongly promotes $\mathrm{G}$ protein bias.

Unsurprisingly scaffold 2 exhibits less receptor diversity than than scaffold 1 . The larger size of scaffold 2 limits a ligands ability to bind to different binding pockets. Indeed, the only receptors targeted by scaffold 2 are D2 and 5-HT1.

In the case of the 5-HT1 receptor, a basic amine appears to play a crucial role for agonist activity. ${ }^{15}$ For scaffold 2, this amine is the aromatic nitrogen. This nitrogen binds to ASP 116 in the $5 \mathrm{HT} 1 \mathrm{~A}$ receptor. ${ }^{16}$ The position of this basic nitrogen is critical for $5 \mathrm{HT} 1 \mathrm{~A}$ agonism. Because of this, it is logical to consider whether the piperazine attached to the pyridine 
is necessary to maintaining an optimal interaction with ASP 116. The correct position of these two sub scaffolds might be critical for maintaining agonism. This idea is supported by the fact that there were no alterations to the 1-(2-Pyridinyl)piperazine backbone for all compounds in the work done by Stroth et al. ${ }^{15}$ Although there is no specific mention of how piperazine interacts with a particular region of the 5HT1A receptor in the work done by Stroth et al., the fact that all of the Stroth scaffolds retain an unchanged piperazine moiety suggest it is crucial for biased activity.

Next, we consider the D2 receptor. G protein biased D2 ligands contain scaffold 2. Unlike the case for the 5HT1A receptor, the literature provides evidence that the basic nitrogen of the piperazine ring together with the aromatic character of the pyridine form a scaffold necessary for biased signaling. Modifying structures adjacent to this scaffold leads to enhanced or diminished biased signaling. ${ }^{17}$ However, the scaffold 2 structure must be left intact for proper signaling.

Scaffold 3 is an ergot alkaloid-like molecule. There are 8 cases of bias on BiasDB with scaffold 3. Of these, 7 cases are associated with Beta-Arrestin bias and 1 case is associated with $\mathrm{G}$ protein selectivity.

Scaffold 3 exhibits a minimal degree of receptor diversity. The scaffold is present in molecules that bind to the $5 \mathrm{HT} 2 \mathrm{~B}$ and $\mathrm{D} 2$ receptors.

The main structural features of this scaffold are a two-ring aromatic moiety and a two ring non-aromatic moiety. Both of these "sides" contain a nitrogen atom which is capable of either donating or accepting a hydrogen bond. The literature does not elaborate on the role the nitrogen atoms have in the binding of $G$ proteins.

In the case of the $5 \mathrm{HT} 2 \mathrm{~B}$ receptor the crucial portion of the molecule responsible for bias is the benzene ring. This ring interacts with LEU 209 on the Extracellular Loop 2 (EL2) motif to prevent ligand egress after binding via hydrophobic interactions. ${ }^{18}$ Prolonged interaction with the 5HT1B receptor is followed by enhanced $\beta$-arrestin recruitment leading to $\beta$-arrestin bias. Similarly for the D2 receptor prolonged interaction of the hydrophobic regions of a 
ligand with ILE 184 leads to enhanced $\beta$-arrestin bias. ${ }^{19}$ Based on this information, the most important portion for bias of scaffold 3 is the benzene ring.

Scaffold 4 is a five member two-nitrogen heterocycle with one double bond. There are 8 cases of bias on BiasDB with scaffold 4. Of these, 4 cases are associated with BetaArrestin bias and the remaining cases are associated with $\mathrm{G}$ protein selectivity or ERK bias. There are no cases of $\mathrm{G}$ protein bias relative to $\beta$-arrestin bias, so we have reason to believe our scaffold is associated with beta-arrestin bias. Scaffold 4 exhibits a minimal degree of receptor diversity on BiasDB. The scaffold is present in molecules that bind to the $1 \mathrm{~A}$ and $2 \mathrm{C}$ adrenoceptors.

Clonodine, moxonidine, and oxymetozaline showed a degree of bias toward $\beta$-Arrestin. ${ }^{20}$ All four of these molecules contained scaffold 4. The work by Kurko et al. demonstrated clonidine, moxonidine, and oxymetazoline had bias factors of 4.28, 3.92, 1.26, respectively . Even though there was no work done to correlate the structural features of scaffold 4 with bias, we suspect the scaffold plays an important role in influencing bias because this moiety was found in all three of these drug molecules. More work needs to be done to elucidate the like between scaffold 4 and $\beta$-arr bias.

Scaffold 5 is a right-hand side bicyclic aromatic moiety. There are 5 cases of bias on BiasDB with this structure. Of these cases, 4 are $\beta$-Arrestin bias and only one $\mathrm{G}$ protein bias. The scaffold is inordinately existing in cases of $\beta$-Arrestin. The literature focuses on the $\mathrm{D} 2-\mathrm{R}$ receptor in vitro and in vivo, giving a holistic approach of these effects. ${ }^{21}$ After synthesizing variations of the bicyclic aromatic moiety, they still exhibited $\beta$-arr bias to varying degrees.

Our reference scaffold, as presented in the literature, differs from molecule 1 which lacks the double bond and 30 which has extra nitrogen in the ring. The Ki value for our scaffold stands at $7.3 \mathrm{nM}$ with a higher inhibition constant in comparison to 1 with $3.9 \mathrm{nM}$ and 30 with $3.4 \mathrm{nM}$. Under the $\beta$-arrestin assay, we have that to reach EC 50; our scaffold molecule needs $6.3 \mathrm{nM}$ compared to 1 and 30 with $4.0 \mathrm{nM}$ and $3.2 \mathrm{nM}$, respectively. Nevertheless, 
when looking at E Max, it has $79 \%$ compared to 1 and 30 that have $62 \%$ and $73 \%$. In retrospect, our scaffold has a higher bias for $\beta$-arr.

In the cAMP assay scaffold 5 has a high EC50 and low EMax compared to molecules 1 and 30, which further confirms the $\beta$-arr bias. It can be inferred that the double bond plays a key role in its bias. The literature further states "Importantly, we observed that subtle structural changes could result in substantial changes in functional selectivity" (Chen et al.,2012)

To explore our results further, we performed an unsupervised machine learning technique, t-stochastic neighbor embedding (t-SNE). We observed similarities between the scaffolds found using t-SNE and those found using supervised learning. In particular we consider three clusters. Cluster 1 (enclosed by a red box in Figure 6) identifies 15 G-protein selective EP2 agonists. These ligands represented in Figure 7 share three features that form a common structural backbone. The SAR of these compounds has previously been described by researchers synthesizing novel EP2 selective agonists. ${ }^{22}$ The first feature is a thiazole ring which is important for EP2 receptor subtype selectivity. ${ }^{23}$ The second is feature is a middle bicyclic moiety which allows the molecules to mimic endogenous prostacyclins. The third feature is a terminal $\omega$ chain which contributes to bias.

Importantly, we note these ligands do not contain secondary amines or aromatic aminesconsistent with the idea that G protein biased ligands tend to have less of these moities than $\beta$-arr biased compounds.

Cluster 2 (enclosed by a yellow box in Figure 6) identifies G-protein selective $\kappa$ opioid receptor agonists. Cluster 2 contains 14 ligands represented in Figure 8. These ligands share a common central triazole which forms a scaffold attached to three different aromatic rings. One of these rings is a 4-pyridyl ring and the remaining two are variable aromatic rings. The SAR of these compounds has previously been described by researchers investigating biased $\kappa$ opioid receptor agonists. ${ }^{13,24 ?}$ To alter bias, the non-pyridyl rings are exchanged but the core triazole ring is left intact. 
The core triazole contains neither aromatic amines, nor secondary amines consistent with the idea that G Protein biased ligands have less of these fragments.

Cluster 3 (enclosed by a blue box in Figure 6) identifies $\beta$-arr biased D2 receptor agonists. Cluster 3 contains 17 ligands, 16 of which are represented in Figure 9. Aripiprazole is excluded from this grouping because it is an unbiased reference compound by which biased compounds are compared. The SAR of these compounds has previously been characterized. ${ }^{21,25-27}$ Each of these ligands can be divided into four regions: a "left-hand side (LHS) phenyl ring," "middle cyclic amino moiety," "central linker," and "right-hand side (RHS) bicyclic aromatic moiety." ${ }^{21}$ The regions we will discuss are the RHS bicyclic aromatic moiety and the middle cyclic amino moiety.

The RHS bicyclic aromatic moiety contains one secondary amine. Our machine learning models associate a greater number of secondary amines to $\beta$-arr bias. Indeed, all of the compounds in cluster 3 share this secondary amine. Interestingly, the work done by Chen and colleagues found one compound with two secondary amines in the RHS bicyclic aromatic moiety. ${ }^{21}$ This compound showed an increased in beta arrestin bias relative to aripiprazole, supporting the connection between number of secondary amines and bias. 


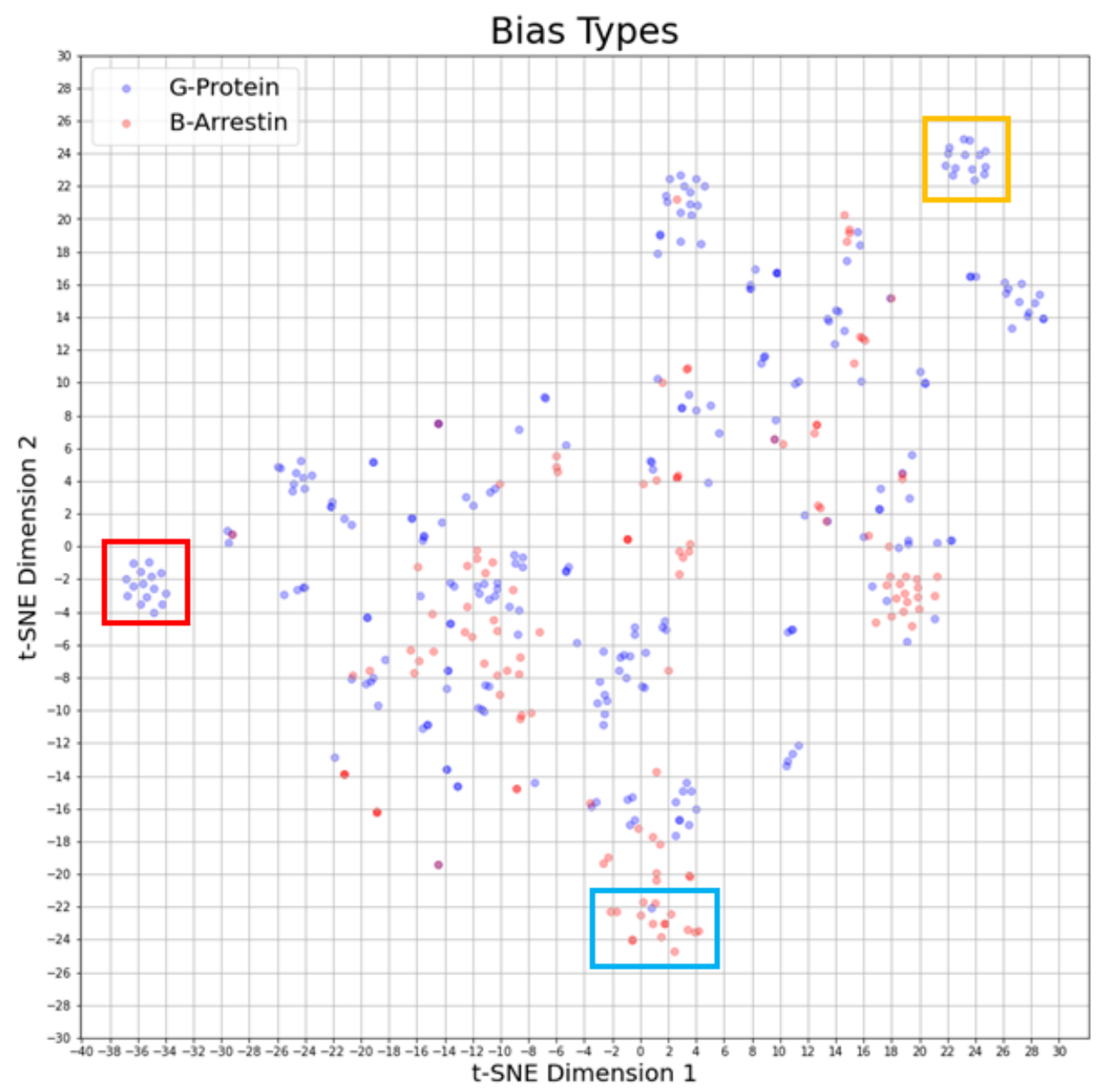

Figure 6: t-SNE clustering. Cluster 1 (red), Cluster 2 (yellow), Cluster 3 (blue). 


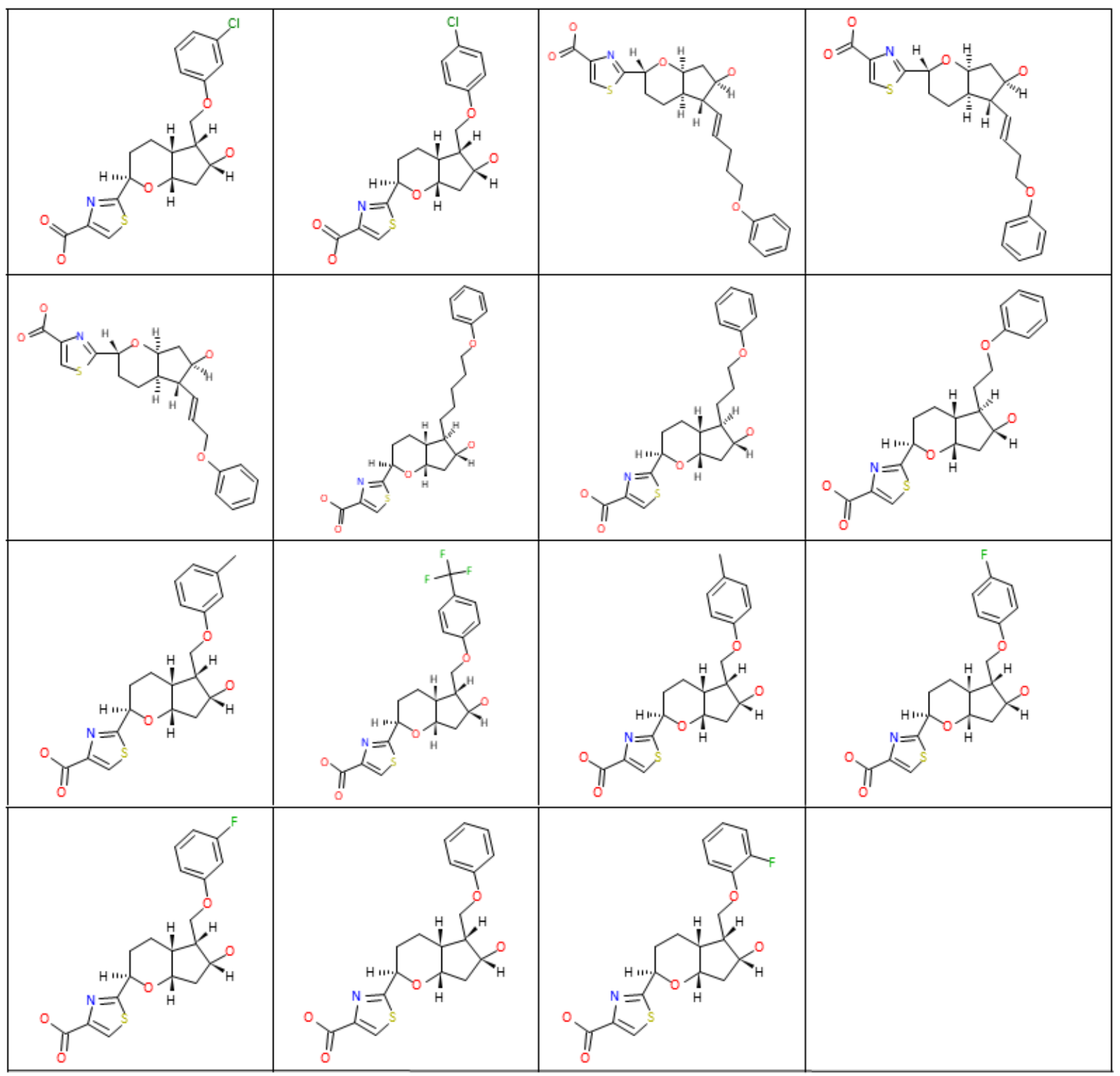

Figure 7: t-SNE Cluster 1 


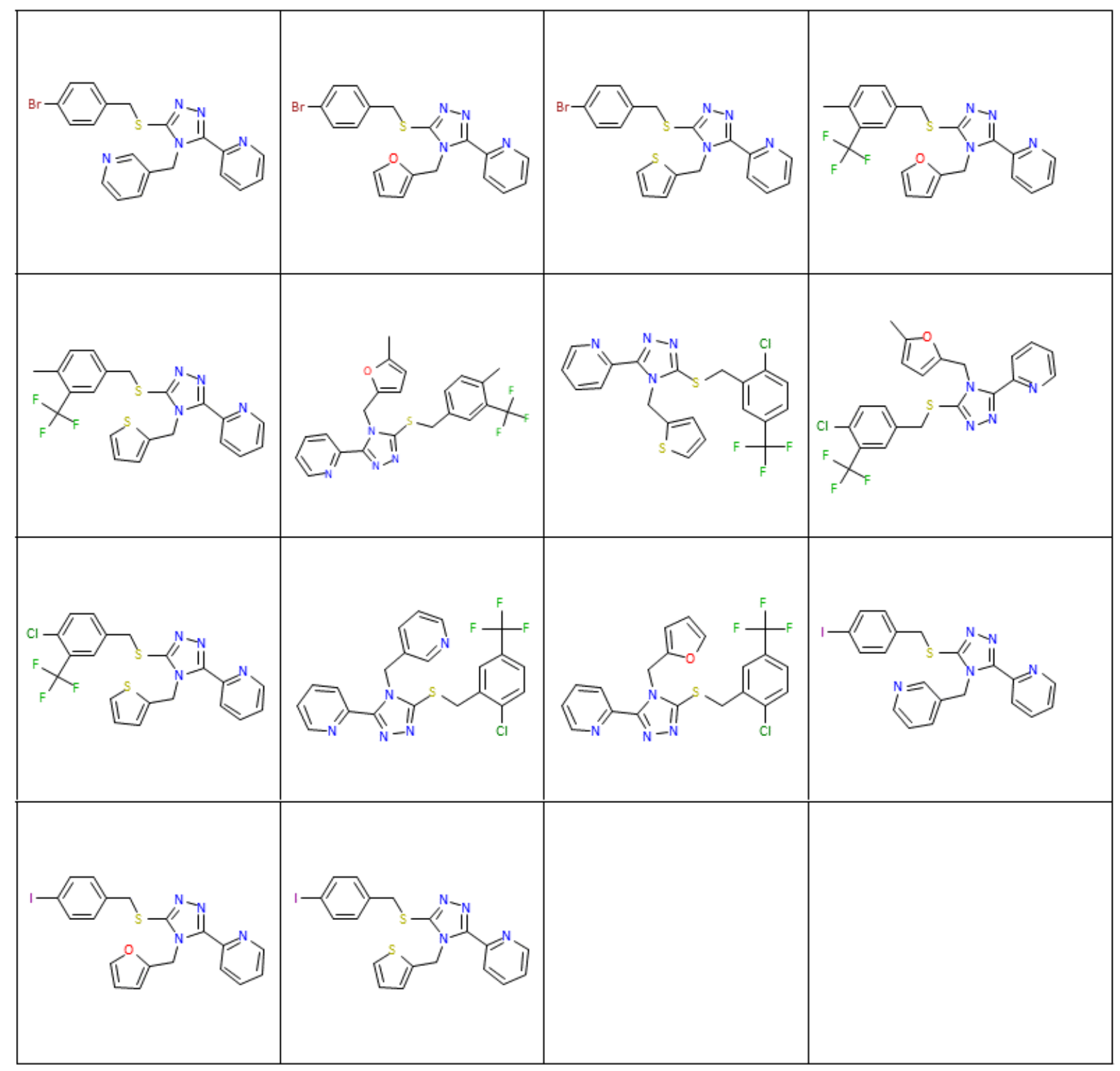

Figure 8: t-SNE Cluster 2 


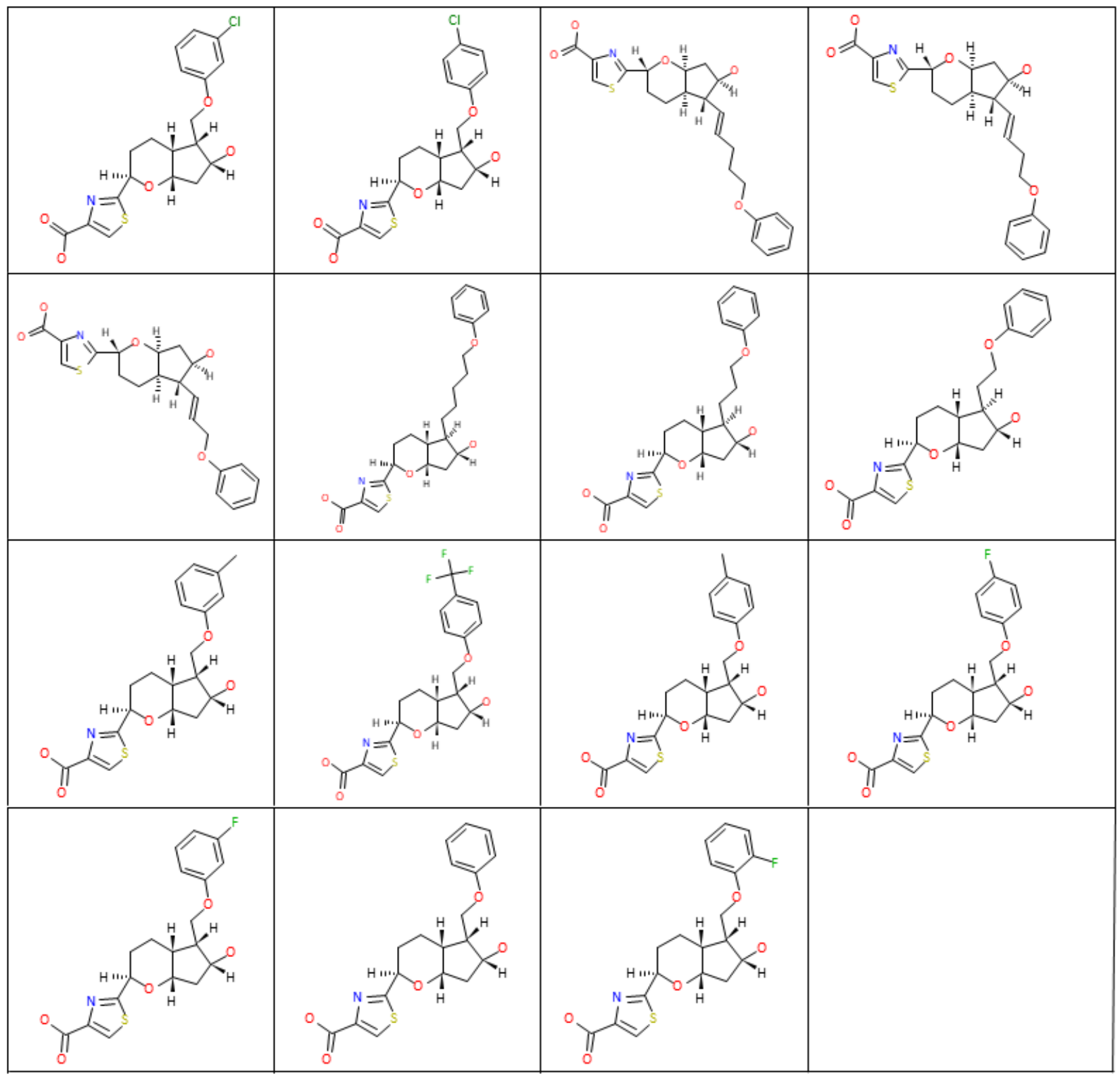

Figure 9: t-SNE Cluster 3 
To end our discussion of scaffolds, we chose to illustrate how bias can have clinically significant effect. Here we consider commercially available medications or drugs undergoing clinical trials.

Oliceridine is a G protein biased drug molecule containing scaffold 1 currently undergoing clinical trials. Oliceridine is a $\mu$-opiod receptor agonist intended to treat severe acute pain. $\beta$-arrestin activity for $\mu$-opiod receptors is associated with increased gastrointestinal dysfunction, respiratory dysfunction and decreased efficacy. ${ }^{28}$ Because Oliceridine shows a marked G protein bias, researchers suspect Oliceridine might have a better safety profile than commercially-available drugs like morphine. Despite this optimism, other researchers suggest the enhanced safety profile of Oliceridine might be attributed to a lower intrinsic activity for the drug. ${ }^{29}$ In any case, more research is needed to determine whether Oliceridine is a suitable alternative for current opiod drugs.

Lisuride is a $\beta$-arr biased drug containing scaffold 3. Lisuride binds to D2 receptors and is indicated for Parkinson's Disease. Standard treatments for Parkinson's include the drug LDopa which activate dopamine receptors in the body. L-Dopa is associated with side effects such as dyskenisia which are thought to be caused by receptor super-sensitivity. ${ }^{30}$ Lisuride bypasses such side effects because its $\beta$-arr action mitigates this receptor super-sensitivity. For this reason, lisuride is often prescribed before L-Dopa.

Clonidine is a $\beta$-arr biased drug containing scaffold 4 . Clonidine binds to the $\alpha 2$ adrenergic receptor and is indicated for for high blood pressure. Work has been conducted for a related groups of anti-hypertensive and cardio-protective drugs targeting the $\beta 1$ adrenergic receptors. This work links $\beta$-arr activation to "therapeutically advantageous" activity. ${ }^{31} \mathrm{Al}-$ though clonidine works through a different receptor and overall mechanism of action, there is reason to believe $\beta$-arr bias for clonidine could also be a desirable characteristic for the drug. For clonidine, $\beta$-arr bias is liked to increased endocytosis of the $\alpha 2$ adrenergic receptor. ${ }^{32}$ This $\beta$-arr directed effect presumably leads to desensitization of the ligand which results in decreased drug side effects. 

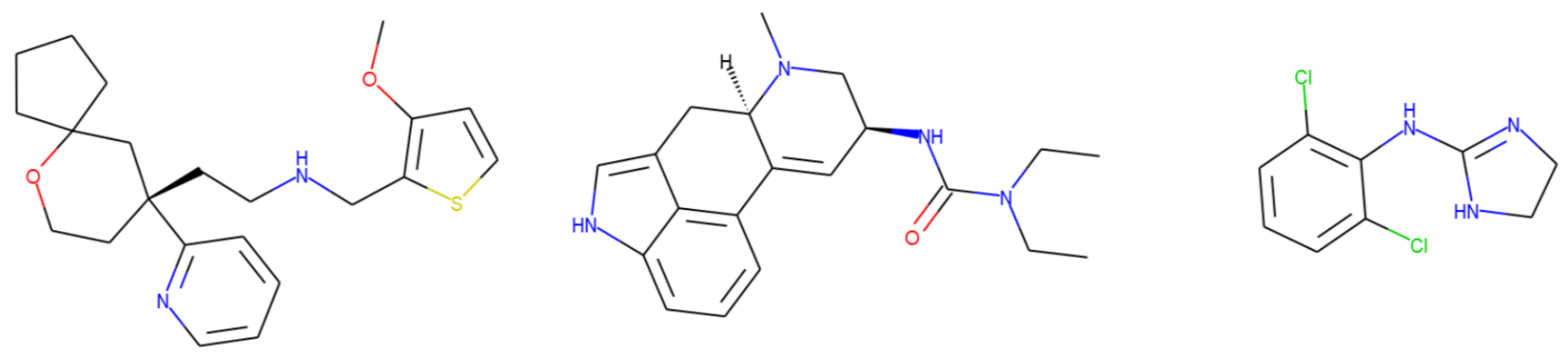

Figure 10: Biased Drug Molecules

\section{Conclusions}

In summary, our work was geared toward determining if there are global patterns of bias among ligands present in the recently available database BiasDB. BiasDB contains cases of bias for many different receptor types and many different ligand types all with different SARs. Our machine learning models accurately picked up on these global patterns and correctly categorized ligands as $\mathrm{G}$ protein biased ligands or $\beta$-arr biased ligands therefore we had reason to believe there likely exists chemical scaffolds which can be used to make these judgments by observation. Moreover such scaffolds can serve as inspiration for future drug development. We employed a machine learning model to find such scaffolds and described the chemistry underlying the some potential examples of structures responsible for global bias. We ended with a discussion of three drug molecules which bear these scaffolds to gain an appreciation for why biased signaling is important.

\section{Methods}

\section{Machine Learning}

The main data set was divided into training (80\%) and test (20\%) sets using stratified sampling method, which ensures the equal distribution of different classes into both the sets. Four different machine learning (ML) algorithms namely, Random Forest, Extreme Gradient Boosting, Multilayer Perceptron (MLP), and Direct Message Passing Neural Network 
(DMPNN) were used for model training. Firstly, all ML algorithms with their default settings were trained with 19 different fingerprints (see Molecular features) and the best models from each classifier (RF, XGB, MLP, and DMPNN) were further tuned based on hyperparmeter optimization. For classical models, we used Hyperopt (Ref) where each model was tuned based on grid search method with default 3 fold cross validation. In order to compare the model performances between the models trained with manually computed molecular fingerprints and models trained on graph based neural networks that learns by operating on graph structure of the molecule, we retrained the models using Chemprop D-MPNN. The DMPNN model was also trained with 3 -fold cross-validation. It was optimized using various model hyperparameters such as: hidden size of the neural network layers, model's depth, numberof feed forward network layers, and dropout probability. Among all the optimized models, the outperforming model based on test set F1 score was used for web implementation. Four aforementioned ML algorithms are summarized as follows.

Random Forest: Random forest is a supervised learning algorithm used for both classification and regression problems. It comprised of multiple decision trees with the technique called bootstrap aggregation (also known as bagging) and gets prediction from each tree outputting the best solution by means of voting. The model was tuned with mainly three hyperparameters (n_estimators, min_samples_split, and max_features) to get the optimized model.

XGBoost: It is also a decision-tree-based ensemble algorithm that uses gradient boosting framework designed for speed and performance. The training of the model can be parallelized/distributed across the clusters. The output of the model is obtained by summing up a sequence of decision trees, called boosting. Each new tree corrects errors which were made by previously trained decision tree. The best model was achieved tuning three different hyperparameters (n_estimators, learning_rate, and colsample_bytree).

Multi-layer Perceptron: Multi-layer Perceptron (MLP) is a feedforward artificial neural network (ANN) capable of mapping the input data into appropriate outputs. ANNs are the 
brain-inspired systems comprised of artificial neurons commonly known as nodes. A MLP consists of input layer, hidden layers, and output layer and each layer is fully connected to the following layer. Each neuron is applied to an activation function except for the input layer. We used a rectified linear unit (ReLU) as an activation function and 'adam' as a solver or weight optimization.

Direct Message Passing Neural Network (D-MPNN) The Direct Message Passing Neural Network (D-MPNN) ${ }^{33}$ is a graph based neural network built on framework of MPNN by Glimer et. al. ${ }^{34}$ An MPNN is a unified framework to generalize several graph convolution neural network methods. The input to MPNN is an undirected graph G with node features $x_{v}$ and bond features $e_{v w}$, where $v$ and $w$ are the node indices. The framework consists of two phases: message passing phase and readout phase. The message passing phase gathers the structural information of the graph and readout phase uses that information of the graph to make predictions. The D-MPNN differs with MPNN in terms of message passing through the network. DMPNN updates the information of the directed bonds where as MPNN updates the information of atoms. We compared the results obtained from the D-MPNN and traditional machine learning model Random forest (RF) using publicly available GitHub repository "Chemprop". ${ }^{33}$

\section{Machine Learning Model Comparison}

Our Machine learning models were evaluated by Receiver Operation Characteristic Area Under the Curve (ROC AUC), Accuracy, F1 Score, Recall, Precision, Sensitivity, Specificity, and Cohen Kappa values. Additionally, a pair-wise McNemar test was conducted to compare the performance of each machine model against all the other models.

\section{Molecular Docking}

All molecular docking experiments were performed using AutoDock Vina version 1.1.2. ${ }^{35}$ The $\beta$-2 Adrenergic Receptor (entry 6KR8) and Dopamine 2 Receptor (entry 6CM4) were 
both obtained from the Protein Data Bank.

\section{Scaffold Selection}

All queried $\mathrm{G}$ protein biased ligands and $\beta$-arr biased ligands were introduced into the hscaf web app available at http://pasilla.health.unm.edu/tomcat/hscaf/hscaf. After processing, 740 scaffolds were generated. These scaffolds were used to develop a RF model for the G protein biased ligands and $\beta$-arr biased ligands.

Following model development, importance scores were calculated using the permutation feature importance module found in Scikit-learn. Scaffolds with a score greater than 0.005 were chosen for screening. Selection based on importance score resulted in 10 scaffolds. Out of these, scaffolds were cross-checked against BiasDB to ensure that the scaffolds represented primarily one type of bias. Five scaffolds fit this criteria.

\section{Acknowledgement}

The authors thank Jeremy Yang from the Translational Informatics Division at the University of New Mexico (UNM) School of Medicine for helping our team implement hscaf and Hassan Mahmudulla for providing support for machine learning model development and web hosting.

\section{Funding}

Dr. Sirimulla acknowledges support from the National Science Foundation through NSFPREM grant DMR-1827745. 


\section{References}

(1) Sriram, K.; Insel, P. A. G protein-coupled receptors as targets for approved drugs: How many targets and how many drugs? Molecular Pharmacology 2018, 93, 251-258.

(2) Hauser, A. S.; Attwood, M. M.; Rask-Andersen, M.; Schiöth, H. B.; Gloriam, D. E. Trends in GPCR drug discovery: New agents, targets and indications. Nature Reviews Drug Discovery 2017, 16, 829-842.

(3) Tan, L.; Yan, W.; McCorvy, J. D.; Cheng, J. Biased Ligands of G Protein-Coupled Receptors (GPCRs): Structure-Functional Selectivity Relationships (SFSRs) and Therapeutic Potential. 2018.

(4) Reiter, E.; Ahn, S.; Shukla, A. K.; Lefkowitz, R. J. Molecular Mechanism of $\beta$-ArrestinBiased Agonism at Seven-Transmembrane Receptors. Annual Review of Pharmacology and Toxicology 2012, 52, 179-197.

(5) Kenakin, T. Agonist-receptor efficacy II: agonist trafficking of receptor signals. Trends in Pharmacological Sciences 1995, 16, 232-238.

(6) Omieczynski, C.; Nguyen, N.; Sribar, D.; Deng, L.; Stepanov, D.; Schaller, D.; Wolber, G.; Bermudez, M. BiasDB: A Comprehensive Database for Biased GPCR Ligands.

(7) Wilkens, S. J.; Janes, J.; Su, A. I. HierS: Hierarchical scaffold clustering using topological chemical graphs. Journal of Medicinal Chemistry 2005, 48, 3182-3193.

(8) Mandrekar, J. N. Receiver operating characteristic curve in diagnostic test assessment. Journal of Thoracic Oncology 2010, 5, 1315-1316.

(9) Weiss, D. R.; Ahn, S.; Sassano, M. F.; Kleist, A.; Zhu, X.; Strachan, R.; Roth, B. L.; Lefkowitz, R. J.; Shoichet, B. K. Conformation guides molecular efficacy in docking screens of activated $\beta-2$ adrenergic G protein coupled receptor. ACS Chemical Biology 2013, 8, 1018-1026. 
(10) Peterson, S. M.; Pack, T. F.; Wilkins, A. D.; Urs, N. M.; Urban, D. J.; Bass, C. E.; Lichtarge, O.; Caron, M. G. Elucidation of G-protein and $\beta$-arrestin functional selectivity at the dopamine D2 receptor. Proceedings of the National Academy of Sciences of the United States of America 2015, 112, 7097-7102.

(11) Männel, B.; Hübner, H.; Möller, D.; Gmeiner, P. $\beta$-Arrestin biased dopamine D2 receptor partial agonists: Synthesis and pharmacological evaluation. Bioorganic and Medicinal Chemistry 2017, 25, 5613-5628.

(12) Gray, D. L.; Allen, J. A.; Mente, S.; O’connor, R. E.; Demarco, G. J.; Efremov, I.; Tierney, P.; Volfson, D.; Davoren, J.; Guilmette, E.; Salafia, M.; Kozak, R.; Ehlers, M. D. Impaired $\beta$-arrestin recruitment and reduced desensitization by non-catechol agonists of the D1 dopamine receptor. Nature Communications 2018, 9.

(13) Zhou, L.; Lovell, K. M.; Frankowski, K. J.; Slauson, S. R.; Phillips, A. M.; Streicher, J. M.; Stahl, E.; Schmid, C. L.; Hodde, P.; Madoux, F.; Cameron, M. D.; Prisinzano, T. E.; Aubé, J.; Bohn, L. M. Development of functionally selective, small molecule agonists at kappa opioid receptors. Journal of Biological Chemistry 2013, 288, 3670336716.

(14) Chen, X. T.; Pitis, P.; Liu, G.; Yuan, C.; Gotchev, D.; Cowan, C. L.; Rominger, D. H.; Koblish, M.; Dewire, S. M.; Crombie, A. L.; Violin, J. D.; Yamashita, D. S. Structureactivity relationships and discovery of a $\mathrm{g}$ protein biased $\mu$ opioid receptor ligand, [(3-methoxythiophen-2-yl)methyl](\{2-[(9 r)-9-(pyridin-2-yl)-6-oxaspiro-[4.5]decan9-yl]ethyl\})amine (TRV130), for the treatment of acute severe pain. Journal of Medicinal Chemistry 2013, 56, 8019-8031.

(15) Stroth, N.; Niso, M.; Colabufo, N. A.; Perrone, R.; Svenningsson, P.; Lacivita, E.; Leopoldo, M. Arylpiperazine agonists of the serotonin 5-HT1A receptor preferentially 
activate cAMP signaling versus recruitment of $\beta$-arrestin-2. Bioorganic and Medicinal Chemistry 2015, 23, 4824-4830.

(16) Ho, B. Y.; Karschin, A.; Branchek, T.; Davidson, N.; Lester, H. A. The role of conserved aspartate and serine residues in ligand binding and in function of the 5-HT1A receptor: A site-directed mutation study. FEBS Letters 1992, 312, 259-262.

(17) Free, R. B. et al. Discovery and characterization of a G protein-biased agonist that inhibits $\beta$-arrestin recruitment to the D2 dopamine receptor. Molecular Pharmacology 2014, 86, 96-105.

(18) Wacker, D.; Wang, S.; McCorvy, J. D.; Betz, R. M.; Venkatakrishnan, A. J.; Levit, A.; Lansu, K.; Schools, Z. L.; Che, T.; Nichols, D. E.; Shoichet, B. K.; Dror, R. O.; Roth, B. L. Crystal Structure of an LSD-Bound Human Serotonin Receptor. Cell 2017, 168, 377-389.e12.

(19) McCorvy, J. D.; Butler, K. V.; Kelly, B.; Rechsteiner, K.; Karpiak, J.; Betz, R. M.; Kormos, B. L.; Shoichet, B. K.; Dror, R. O.; Jin, J.; Roth, B. L. Structure-inspired design of $\beta$-arrestin-biased ligands for aminergic GPCRs. Nature Chemical Biology 2018, 14, $126-134$.

(20) Kurko, D.; Kapui, Z.; Nagy, J.; Lendvai, B.; Kolok, S. Analysis of functional selectivity through G protein-dependent and -independent signaling pathways at the adrenergic a2C receptor. Brain Research Bulletin 2014, 10\%, 89-101.

(21) Chen, X.; Sassano, M. F.; Zheng, L.; Setola, V.; Chen, M.; Bai, X.; Frye, S. V.; Wetsel, W. C.; Roth, B. L.; Jin, J. Structure-functional selectivity relationship studies of $\beta$-arrestin-biased dopamine D2 receptor agonists. Journal of Medicinal Chemistry 2012, 55, 7141-7153.

(22) Ogawa, S.; Watanabe, T.; Sugimoto, I.; Moriyuki, K.; Goto, Y.; Yamane, S.; Watanabe, A.; Tsuboi, K.; Kinoshita, A.; Kigoshi, H.; Tani, K.; Maruyama, T. Discovery of 
G Protein-Biased EP2 Receptor Agonists. ACS Medicinal Chemistry Letters 2016, 7, 306-311.

(23) Kambe, T.; Maruyama, T.; Nakai, Y.; Yoshida, H.; Oida, H.; Maruyama, T.; Abe, N.; Nishiura, A.; Nakai, H.; Toda, M. Discovery of novel prostaglandin analogs as potent and selective EP2/EP4 dual agonists. Bioorganic \& Medicinal Chemistry 2012, 20, 2235-2251.

(24) M. Lovell, K.; J. Frankowski, K.; L. Stahl, E.; R. Slauson, S.; Yoo, E.; E. Prisinzano, T.; Aubé, J.; M. Bohn, L. Structure-Activity Relationship Studies of Functionally Selective Kappa Opioid Receptor Agonists that Modulate ERK 1/2 Phosphorylation While Preserving G Protein Over $\beta$ Arrestin2 Signaling Bias. ACS Chemical Neuroscience 2015, $6,1411-1419$.

(25) Vangveravong, S.; Zhang, Z.; Taylor, M.; Bearden, M.; Xu, J.; Cui, J.; Wang, W.; Luedtke, R. R.; MacH, R. H. Synthesis and characterization of selective dopamine D2 receptor ligands using aripiprazole as the lead compound. Bioorganic and Medicinal Chemistry 2011, 19, 3502-3511.

(26) Oshiro, Y.; Sato, S.; Kurahashi, N.; Tanaka, T.; Kikuchi, T.; Tottori, K.; Uwahodo, Y.; Nishi, T. Novel antipsychotic agents with dopamine autoreceptor agonist properties: Synthesis and pharmacology of 7-[4-(4-phenyl-1- piperazinyl)butoxy]-3,4dihydro-2(1H)-quinolinone derivatives. Journal of Medicinal Chemistry 1998, 41, 658667.

(27) Johnson, D. S.; Choi, C.; Fay, L. K.; Favor, D. A.; Repine, J. T.; White, A. D.; Akunne, H. C.; Fitzgerald, L.; Nicholls, K.; Snyder, B. J.; Whetzel, S. Z.; Zhang, L.; Serpa, K. A. Discovery of PF-00217830: Aryl piperazine napthyridinones as D2 partial agonists for schizophrenia and bipolar disorder. Bioorganic and Medicinal Chemistry Letters. 2011; pp 2621-2625. 
(28) DeWire, S. M.; Yamashita, D. S.; Rominger, D. H.; Liu, G.; Cowan, C. L.; Graczyk, T. M.; Chen, X. T.; Pitis, P. M.; Gotchev, D.; Yuan, C.; Koblish, M.; Lark, M. W.; Violin, J. D. A G protein-biased ligand at the $\mu$-opioid receptor is potently analgesic with reduced gastrointestinal and respiratory dysfunction compared with morphines. Journal of Pharmacology and Experimental Therapeutics 2013, 344, 708-717.

(29) Gillis, A. et al. Low intrinsic efficacy for G protein activation can explain the improved side effect profiles of new opioid agonists. Science Signaling 2020, 13.

(30) Urs, N. M.; Bido, S.; Peterson, S. M.; Daigle, T. L.; Bass, C. E.; Gainetdinov, R. R.; Bezard, E.; Caron, M. G. Targeting $\beta$-arrestin2 in the treatment of L-DOPA-induced dyskinesia in Parkinson's disease. Proceedings of the National Academy of Sciences of the United States of America 2015, 112, E2517-E2526.

(31) Carr, R.; Schilling, J.; Song, J.; Carter, R. L.; Du, Y.; Yoo, S. M.; Traynham, C. J.; Koch, W. J.; Cheung, J. Y.; Tilley, D. G.; Benovic, J. L. $\beta$-arrestin-biased signaling through the $\beta 2$-adrenergic receptor promotes cardiomyocyte contraction. Proceedings of the National Academy of Sciences of the United States of America 2016, 113, E4107E4116.

(32) Lu, R.; Li, Y.; Zhang, Y.; Chen, Y.; Shields, A. D.; Winder, D. G.; Angelotti, T.; Jiao, K.; Limbird, L. E.; Zhou, Y.; Wang, Q. Epitope-tagged receptor knock-in mice reveal that differential desensitization of $\alpha 2$-adrenergic responses is because of ligandselective internalization. Journal of Biological Chemistry 2009, 284, 13233-13243.

(33) Yang, K.; Swanson, K.; Jin, W.; Coley, C.; Eiden, P.; Gao, H.; Guzman-Perez, A.; Hopper, T.; Kelley, B.; Mathea, M.; Palmer, A.; Settels, V.; Jaakkola, T.; Jensen, K.; Barzilay, R. Analyzing Learned Molecular Representations for Property Prediction. Journal of Chemical Information and Modeling 2019, 59, 3370-3388. 
(34) Gilmer, J.; Schoenholz, S. S.; Riley, P. F.; Vinyals, O.; Dahl, G. E. Neural Message Passing for Quantum Chemistry. 2017.

(35) Trott, O.; Olson, A. J. AutoDock Vina: Improving the speed and accuracy of docking with a new scoring function, efficient optimization, and multithreading. Journal of Computational Chemistry 2009, 31, NA-NA. 